\title{
Myocardial apoptosis associated with the expression of proinflammatory cytokines during the course of myocardial infarction
}

\author{
Yoshikiyo Akasaka ${ }^{1}$, Noriko Morimoto ${ }^{2}$, Yukio Ishikawa ${ }^{1}$, Kazuko Fujita ${ }^{1}$, Kinji Ito ${ }^{1}$, \\ Masayo Kimura-Matsumoto ${ }^{1}$, Shigeki Ishiguro ${ }^{1}$, Hiroshi Morita ${ }^{3}$, Yoshiro Kobayashi ${ }^{2}$ \\ and Toshiharu Ishii ${ }^{1}$
}

${ }^{1}$ Department of Pathology, School of Medicine, Toho University, Tokyo, Japan; ${ }^{2}$ Department of Biomolecular
Science, Faculty of Science, Toho University, Funabashi, Japan and ${ }^{3}$ Department of Gastroentrology and
Hepatology, School of Medicine, Toho University, Tokyo, Japan

To clarify the role of myocardial apoptosis associated with the expression of proinflammatory cytokines in human myocardial infarction (MI), we have analyzed the expression of apoptosis positive for single-stranded DNA (ss-DNA) antibody, tumor necrosis factor (TNF)- $\alpha$, and interleukin (IL)-8 in 147 samples of infarcted myocardial tissue from 65 patients. Ss-DNA-positive apoptotic nuclei were found mainly in cardiomyocytes in the border zones and granulation tissue cells in the infarct foci. The ss-DNA index (SI) of cardiomyocytes (average $0.13 \%$ ) peaked at stage II (established myocardial necrosis), the value being significantly higher than at stages III (macrophage infiltration), IV (granulation formation), and V (scar formation) $(P<0.05)$, whereas the $\mathrm{SI}$ of granulation tissue (average $0.08 \%$ ) at stages III, IV, and V showed no significant differences between the three stages. These results suggest that cardiomyocyte apoptosis in the border zone is responsible for cellular loss in the acute stage of MI, whereas granulation tissue apoptosis may not be involved in the process of ventricular remodeling. TNF- $\alpha$ was expressed in cardiomyocytes in the border zones of infarct foci, but no significant positive correlation was found between $\mathrm{SI}$ and TNF- $\alpha$ index in cardiomyocytes $(r=0.08, P=0.37)$, suggesting that TNF- $\alpha$ does not serve as a direct trigger of cardiomyocyte apoptosis in vivo. The number of IL-8positive cells peaked at stage II, and IL-8-myeloperoxidase-double-positive neutrophils were frequently detected, indicating that infiltrating neutrophils are the predominant source of IL-8 in the infarcted myocardium. These results suggest that, in human MI, TNF- $\alpha$ produced by cardiomyocytes does not play a critical role in their apoptosis, and that IL-8 produced by neutrophils is responsible for the subsequent accumulation and activation of neutrophils, thus increasing the degree of myocardial damage.

Modern Pathology (2006) 19, 588-598. doi:10.1038/modpathol.3800568; published online 3 March 2006

Keywords: apoptosis; cardiomyocyte; granulation tissue; IL-8; TNF- $\alpha$

Apoptosis has been shown to occur in cardiomyocytes during the acute and chronic stages of myocardial infarction (MI) in both animal models and humans. ${ }^{1-6}$ Recent studies have suggested that apoptosis in granulation tissue cells after MI plays an important role in cardiac remodeling during the chronic stages of MI. ${ }^{7,8}$ However, there has been little morphological evidence of apoptosis in both cardiomyocytes and granulation tissue cells occurring at different stages of MI in humans. Furthermore, the rate of cardiomyocyte apoptosis

Correspondence: Dr Y Akasaka, MD, Department of Pathology, School of Medicine, Toho University, 5-21-16 Omori-Nishi, Ohta City, Tokyo 143-8540, Japan.

E-mail: akasakay@med.toho-u.ac.jp

Received 12 September 2005; revised 19 January 2006; accepted 20 January 2006; published online 3 March 2006 in human MI has been reported to vary (0.12$12 \%)^{9,10}$ Apoptotic cells are usually identified by terminal deoxynucleotide transferase-mediated dUTP nick end-labeling (TUNEL). Recently, a single-stranded DNA (ss-DNA) monoclonal antibody (clone F7-26) has been reported to be sufficiently sensitive to detect cells at an incipient stage of apoptosis. ${ }^{11,12}$ Therefore, the present study has examined the proportions of apoptosis in both cardiomyocytes and granulation tissue cells in 147 samples of infarcted myocardial tissue obtained at autopsy from 65 patients using an anti-ss-DNA antibody, and investigate the role of apoptosis in both cardiomyocytes and granulation tissue cells during the course of human MI.

In patients with MI, proinflammatory cytokines, such as tumor necrosis factor (TNF)- $\alpha$ and interleukin (IL)-8 play an important role in triggering 
additional cellular inflammatory responses, and subsequent cytotoxic injury. ${ }^{13,14}$ Previous investigators have speculated that TNF- $\alpha$ might induce apoptosis in cardiomyocytes during acute MI, because of the high serum level of TNF- $\alpha$ in patients with heart failure ${ }^{15,16}$ and the close relationship between the level of TNF- $\alpha$ expression and apoptosis in cardiomyocytes in vitro. ${ }^{17}$ However, there have been few studies on the expression pattern of TNF- $\alpha$ associated with cardiomyocyte apoptosis in human MI. ${ }^{18,19}$ Therefore, the present study has examined the in vivo role of TNF- $\alpha$ in promoting apoptosis in cardiomyocytes by quantitative analysis of the distribution of TNF- $\alpha$ expression and ss-DNA-positive apoptosis in cardiomyocytes in human MI.

It is generally believed that phagocytosis of apoptotic cells by macrophages leads to the production of anti-inflammatory cytokines, such as IL-10, whereas proinflammatory cytokines, such as IL-8, are depleted, thereby preventing immune and inflammatory responses. ${ }^{20,21}$ However, a recent study has revealed that monocytes and monocytederived macrophages release IL-8 in the absence or presence of apoptosis following Fas stimulation. ${ }^{22}$ Another study has shown that Fas induces both apoptosis and IL-8 expression in human glioma cell lines. ${ }^{23}$ With regard to MI, it has been suggested that ischemia in the acute stage creates a proinflammatory environment and that myocardial tissue serves as an important source of IL-8 under such conditions, ${ }^{13,14}$ as it has been demonstrated clinically that the serum level of IL-8 increases transiently in patients during acute MI. ${ }^{24-26}$ However, there has been no direct morphological evidence of the source of IL-8 expression in relation to the distribution of myocardial apoptosis and macrophage infiltration within the infarcted myocardium. ${ }^{25}$ Therefore, the present study has examined the distribution of IL-8-positive cells to reveal the source of IL-8 in human MI.

\section{Materials and methods}

\section{Autopsy Cases}

Samples of myocardial tissue were obtained at autopsy from 65 Japanese patients clinically diagnosed as acute MI or old MI. The patients comprised 18 with acute MI, 22 with old MI, and 25 with both acute MI and old MI. The mean age of the 65 patients was $73.4 \pm 16.7$ years, and there were 38 male and 27 female patients. All the patients were autopsied within $6 \mathrm{~h}$ post mortem. Three samples of normal ventricular tissue without any infarcts, for use as normal controls, were obtained from patients aged 48, 54, and 63 years, who were autopsied within $5 \mathrm{~h}$ of death due to cause other than MI. After the myocardial tissue samples had been excised from the left ventricle and/or ventricular septum, they were fixed in $10 \%$ neutral-buffered formalin and embedded in paraffin. Thin sections were stained with hematoxylin-eosin and azan-Mallory. All cases had infarcted lesions at combined histological stages in the left ventricle and/or ventricular septum. Therefore, we selected two or three myocardial tissue sections in each case, and the average number of sections obtained from one case was 2.3. Finally, we obtained 147 samples of infarcted tissue from the 65 patients, and determined the stages of MI in each section according to the histological criteria for MI described below. Written informed consent was obtained from the patients' families for all procedures, and the study was approved by the Toho University Committee for the Conduct of Human Studies in accordance with the principles of the Declaration of Helsinki.

\section{Classification of MI Stages}

Analysis was performed in one area that showed a maximal infarct size without showing combined stages of MI. In the present study, all infarcted areas observed in one tissue section were divided into five categories based on the sequential histological changes that occur in the healing of MI, according to the Lodge-Patch classification: ${ }^{27,28}$ stage I, the earliest changes represented by stretching and waviness of myocardial fibers, the myocytes showing eosinophilic cytoplasm and pyknosis; stage II, coagulation necrosis of the myocardium with or without pyknotic nuclei, accompanied by neutrophil infiltration; stage III, fragmentation of necrotic cardiomyocytes with macrophage infiltration, and fibroblasts evident in the interstitium; stage IV, replacement of the lesion by granulation tissue with proliferation of fibroblasts, in which the affected cardiomyocytes disappear and neutrophils are decreased; stage V, replacement of the lesion with scar tissue. The 147 specimens of infarcted tissue included 26 stage I lesions, 35 stage II lesions, 26 stage III lesions, 35 stage IV lesions, and 25 stage V lesions.

\section{Immunohistochemistry}

Immunohistochemistry for ss-DNA was carried out using an anti-ss-DNA monoclonal antibody (mAb) (clone F7-26, Chemicon Int. Inc., CA, USA) according to the method as described previously. ${ }^{12}$ Immunohistochemistry was also performed using the following primary antibodies: anti-human CD68 mAb (PG-M1; Dako) at a dilution of 1:200, anti-human TNF- $\alpha$ mAb (clone 28401; Dako) at a dilution of 1:100, anti-human IL-8 polyclonal antibody (pAb) (clone PS038; MONOSAN) at a dilution of 1:50, and anti-human IL-10 (clone MAB217; R\&D Systems) at a dilution of 1:200. The anti-IL-8, IL-10, and CD68 antibodies were incubated for $60 \mathrm{~min}$, and the LSAB2 kit (Dako) was used. To examine the expression pattern of $\mathrm{TNF}-\alpha$, the sections were incubated overnight at $4^{\circ} \mathrm{C}$ in a humidified chamber 
with the anti-TNF- $\alpha$ antibody. Antibody staining was performed with a catalyzed signal amplification kit (DAKO) containing biotinylated anti-mouse IgG $\mathrm{Ab}$ and streptavidin-peroxidase complex. As positive controls for IL-8 and IL-10 staining, the tissue sections from normal lymph nodes and skin with psoriasis were used, respectively. Negative controls were generated by incubating the slides with immunoglobulin from the same species and at the same final concentration, but with no primary antibody.

Double staining for IL-8 and myeloperoxidase was also performed on the sections. After staining using the same protocol as that for IL-8 staining, the sections were incubated overnight at $4{ }^{\circ} \mathrm{C}$ with antihuman myeloperoxidase pAb (A0398, DAKO) at a dilution of 1:300 and then with alkaline phosphatase-conjugated anti-rabbit IgG antibody (Dako) for $1 \mathrm{~h}$. They were then treated with alkaline phosphatase substrate solution (Dako new fuchsin substrate system; Dako) for visualization of the antigens. The negative controls were incubated with rabbit immunoglobulin (X0903, Dako) at the same final concentration, but without the primary antibodies.

\section{Statistical Analysis}

Apoptosis and cytokine expression were analyzed in one infarcted area that showed a maximal size without showing combined stages of MI. For statistical analysis of apoptotic cells and TNF- $\alpha$ positive cells, each specimen stained with the antiss-DNA mAb or the-anti-TNF- $\alpha$ mAb was observed by light microscopy at $\times 200$ magnification.

For stage I and II lesions, three unbiased observers (NM, YI, TI) counted the number of ss-DNA-positive cardiomyocytes and TNF- $\alpha$-positive cardiomyocytes in 10 randomly selected fields in the infarcted tissue, the tissue bordering the infarction, and remote noninfarcted tissue. For stage III lesions, the observers counted the number of ss-DNApositive cardiomyocytes and TNF- $\alpha$-positive cardiomyocytes in 10 randomly selected fields in the border zones of the infarcted areas and in remotely located noninfarcted myocardium. They also counted the number of ss-DNA-positive cells in 10 randomly selected fields in granulation tissue of damaged areas. For stage IV and V lesions, the numbers of ss-DNA-positive cardiomyocytes and TNF- $\alpha$-positive cardiomyocytes were counted in 10 randomly selected fields in the border zones of infarct scars or granulation tissue, and in remotely located noninfarcted myocardium. The observers also counted the number of ss-DNA-positive cells in 10 randomly selected fields in scars or granulation tissue. The cardiomyocyte origin of the apoptotic cells was confirmed by morphological observation, noting especially the presence of myofilaments in the cytoplasm. ${ }^{29}$ According to the levels of ss-DNApositive nuclear staining, three types of staining pattern were mainly observed: unstained, lightly or moderately stained, and strongly stained. For counting, only cells that showed strong nuclear staining were regarded as positive. Inflammatory cells including lymphocytes and macrophages are known to show active proapoptotic interactions through death ligands and receptors. It is therefore speculated that apoptotic mechanisms may be differently regulated in these cell types that may have higher sensitivity to apoptotic stimuli compared with the other cells. ${ }^{7}$ Therefore, small round ss-DNA-positive cells whose origin could not be identified from cardiomyocytes, interstitial cells, or inflammatory cells, were not counted in the statistical analysis. The proportion of ss-DNA-positive cells and TNF- $\alpha$ positive cardiomyocytes was expressed as a ss-DNA index (SI) and TNF- $\alpha$ index (TI) (SI = number of ssDNA-positive cardiomyocytes or granulation tissue cells/total number of cardiomyocytes or granulation tissue cells (approximately 1000 cells per section) $\times 100 \%, \quad \mathrm{TI}=$ number of $\mathrm{TNF}-\alpha$-positive cardiomyocytes/total number of cardiomyocytes (approximately 1000 cells per section) $\times 100 \%$ ).

The numbers of IL-8-positive cells were counted in 10 randomly selected high-power fields at $\times 400$ magnification and the result was expressed as an IL8 index (IL-8 index = number of IL-8-positive cells/ total number of inflammatory cells (approximately 800 cells per section) $\times 100 \%$ ).

The number of IL-10 or CD68-positive cells was similarly counted in 10 randomly selected fields at $\times 40$ magnification, and used to calculate the mean number of positively stained cells per microscopic field.

All results were expressed as the mean \pm standard errors of the mean and statistical analysis was conducted using an analysis of variance (ANOVA) followed by a Bonferroni's comparison test using the Statview-J 4.5 software package (Abacus Concepts Inc., Berkeley, CA, USA). Results were considered to be statistically significant at $P<0.05$.

\section{Results}

\section{SsDNA-Positive Apoptotic Cells during the Course of MI}

Absence of ss-DNA-positive cells was confirmed in normal control myocardium. In the foci of stage I lesions, thinning and waviness of myocardial fibers was evident, and nuclei were distinctly visible (Figure 1a). ss-DNA-positive nuclei were found only in cardiomyocytes in the infarcted areas (Figure 1b). In some cases, a very small number of ss-DNA-positive nuclei were also found in the interstitium.

In stage II lesions, most cardiomyocytes exhibited coagulation necrosis, in which their cytoplasm showed an eosinophilic hue with or without pyknotic nuclei (Figure 1c). ss-DNA-positive apoptosis was detected mainly in noninfarcted 

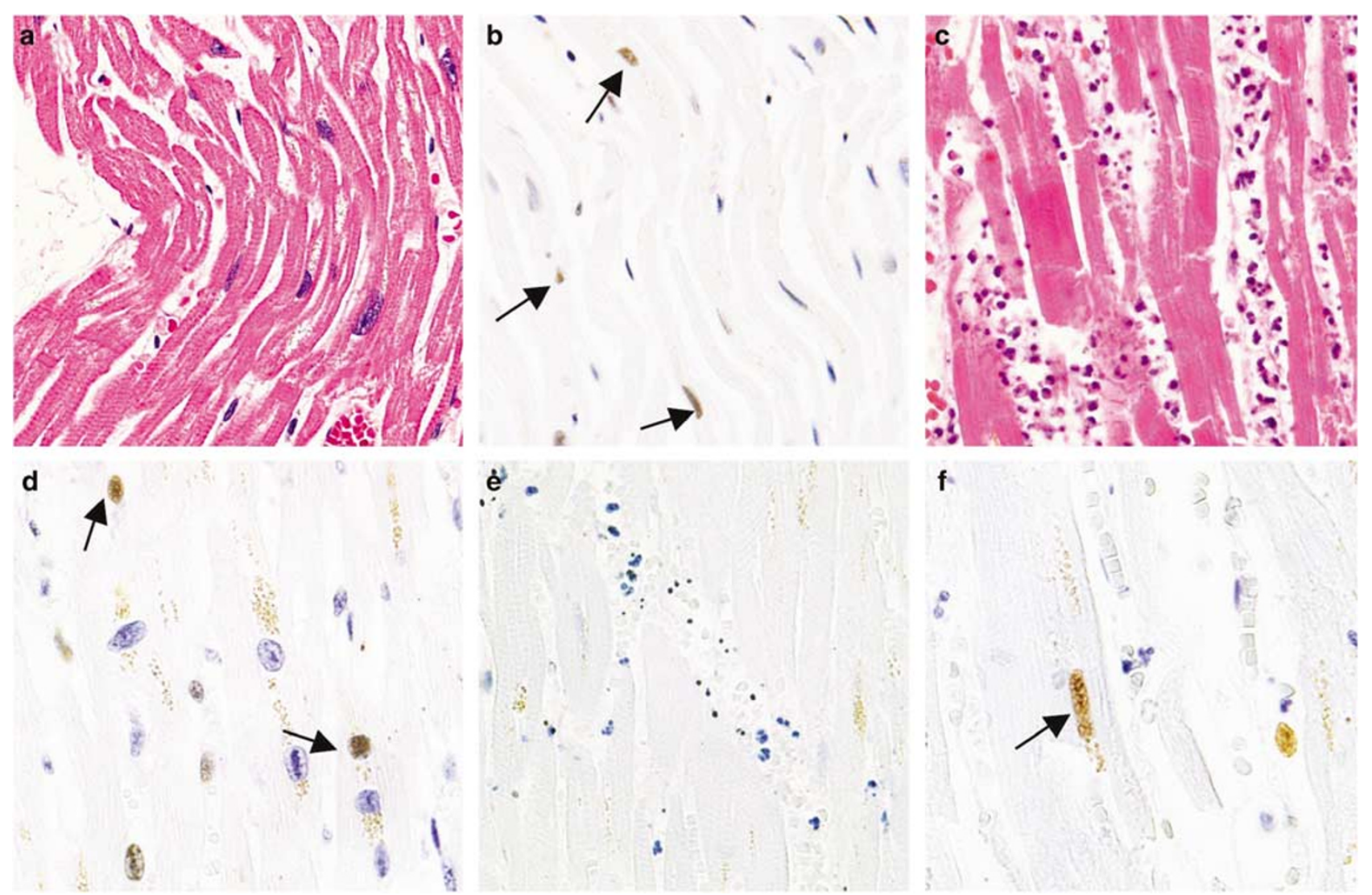

Figure 1 ss-DNA-positive apoptotic cells at stage I (early myocardial infarction: a, b) and stage II (established myocardial necrosis: c-f). (a) The stage I infarct focus shows thinning and waviness of damaged myocardial fibers. Hematoxylin-eosin staining. (b) ss-DNApositive nuclei are evident in the infarcted cardiomyocytes (arrows), which have become smaller and show condensed chromatin, indicating morphological change due to apoptosis. (c) The stage II infarct focus shows cardiomyocytes with coagulation necrosis and marked infiltration of neutrophils in the interstitium. Hematoxylin-eosin staining. (d) ss-DNA-positive cells in the noninfarcted cardiomyocytes in the border zone. The positive nuclei (arrows) have become smaller, suggesting morphological change due to apoptosis. (e) ssDNA-positive cells are absent in the infarcted focus. (f) A ss-DNA-positive cell (arrow) is evident in the infarcted cardiomyocytes.

cardiomyocytes in the border zones of the infarct (Figure 1d). ss-DNA-positive apoptotic cells were not almost found in infarcted cardiomyocytes (Figure 1e). However, in some cases, a very small number of positive nuclei were found in infarcted cardiomyocytes within the infarct (Figure 1f).

In stage III lesions, the cardiomyocytes with coagulation necrosis were fragmented, and many macrophages were present in granulation tissue of damaged areas (Figure 2a). ss-DNA-positive nuclei were found mainly in fibroblasts in area of granulation tissue (Figure 2b). Scattered ss-DNA-positive cells were also found in cardiomyocytes in the border zone (Figure 2c) or in remotely located noninfarcted cardiomyocyte (Figure 2d), the number of positive cells decreasing markedly with distance from the damaged focus.

In stage IV lesions, the infarct focus was replaced with granulation tissue with an increased number of fibroblasts (Figure 2e). A few scattered ss-DNApositive cells were found in the fibroblasts within areas of granulation tissue (Figure 2f) or in cardiomyocytes in the border zone of the damaged focus.
Stage V lesions showed replacement with scar tissue (Figure 2g). A few scattered ss-DNA-positive cardiomyocytes were found in the border zone of the infarct scar (Figure 2h). In some cases, very few scattered ss-DNA-positive fibroblasts were also evident in the infarct scar.

\section{Expression of TNF- $\alpha$, IL-8, IL-10, and CD68 during the Course of MI}

Absence of TNF- $\alpha$-positive cells was confirmed in normal control myocardium. In stage I lesions, IL-8 expression was found in very few scattered neutrophils infiltrating in the interstitium around noninfarcted cardiomyocytes (Figure 3a). TNF- $\alpha$ expression was observed in some noninfarct cardiomyocytes.

In stage II lesions, infiltration of neutrophils was detected more frequently in the damaged focus compared with stage I lesions. IL-8-positive cells were intermingled with the neutrophils infiltrating the infarct focus, and double staining for IL-8 and 

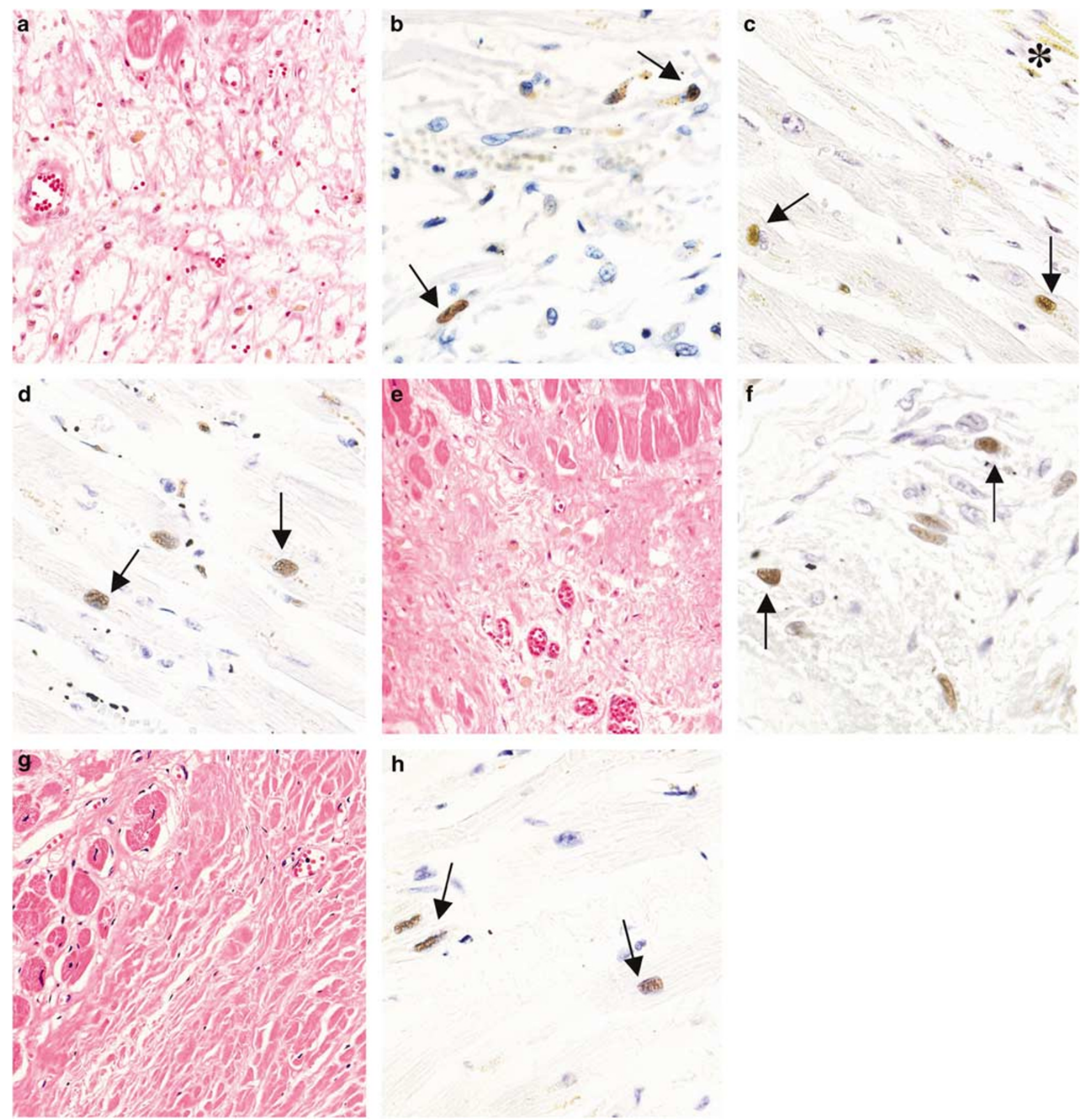

Figure 2 ss-DNA-positive apoptotic cells at stage III (macrophage infiltration: a-d), stage IV (granulation formation: e, f), and stage V (scar formation: g, h). (a) The stage III infarct focus contains many macrophages, and also a few fibroblasts with fragmented cardiomyocytes. Hematoxylin-eosin staining. (b) In the granulation tissue of the damaged area, ss-DNA-positive nuclei are evident in the fibroblasts (arrows). (c) ss-DNA-positive cardiomyocytes (arrows) in the border zone. The upper half of the figure $\left({ }^{*}\right)$ shows the granulation tissue. (d) ss-DNA-positive cardiomyocytes (arrows) in an area remote from the infarcted myocardium. (e) A stage IV lesion showing replacement with granulation tissue. Hematoxylin-eosin staining. (f) ss-DNA-positive nuclei are evident in fibroblasts (arrows) within an area of granulation tissue. (g) A stage V lesion showing replacement with scar tissue. (h) ss-DNA-positive cardiomyocytes (arrows) in the border zone of the infarct scar.

myeloperoxidase confirmed that neutrophils were positive for both (Figure 3b-e). TNF- $\alpha$ expression was found in cardiomyocytes in the border zone or in the vicinity of the infarct focus (Figure 3f), whereas its expression was not observed in cardiomyocytes within the infarct (Figure 3g).
In stage III lesions, along with an increased number of macrophages, CD68-positive cells were frequently observed in granulation tissue. IL-10positive cells were scattered in some parts of the interstitium around noninfarcted cardiomyocytes (Figure 3h). Similar to the expression pattern 

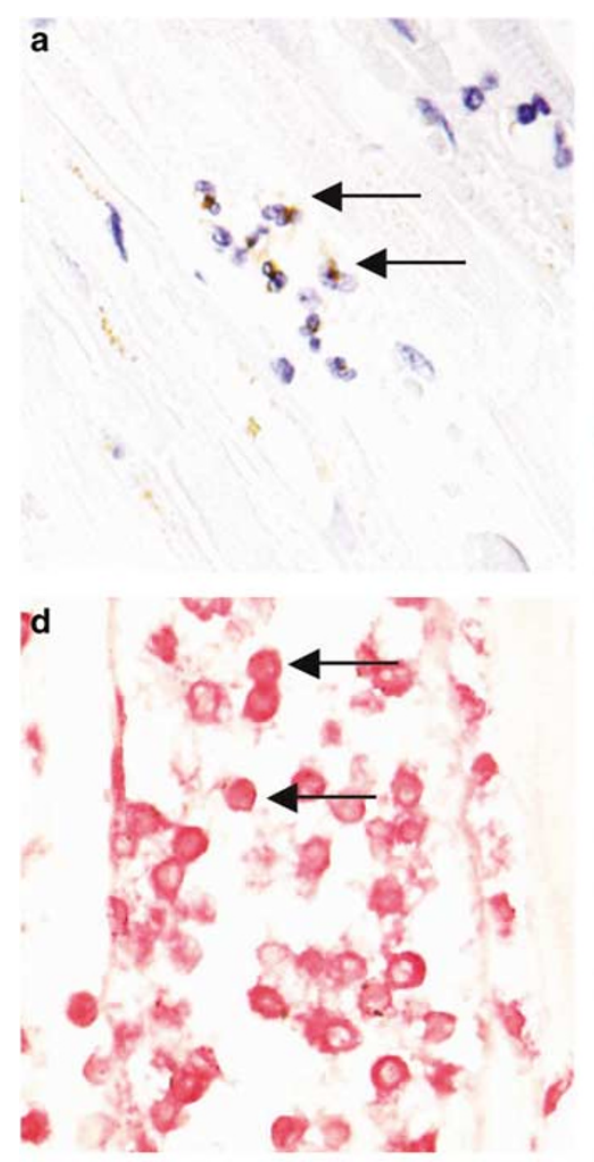

g
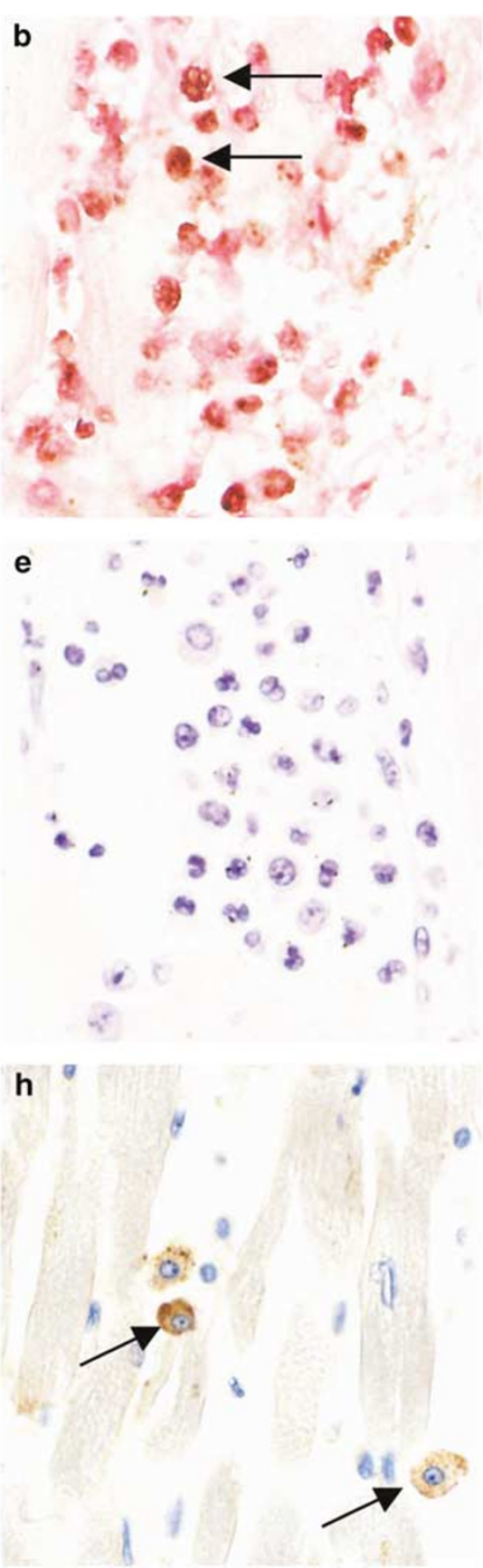
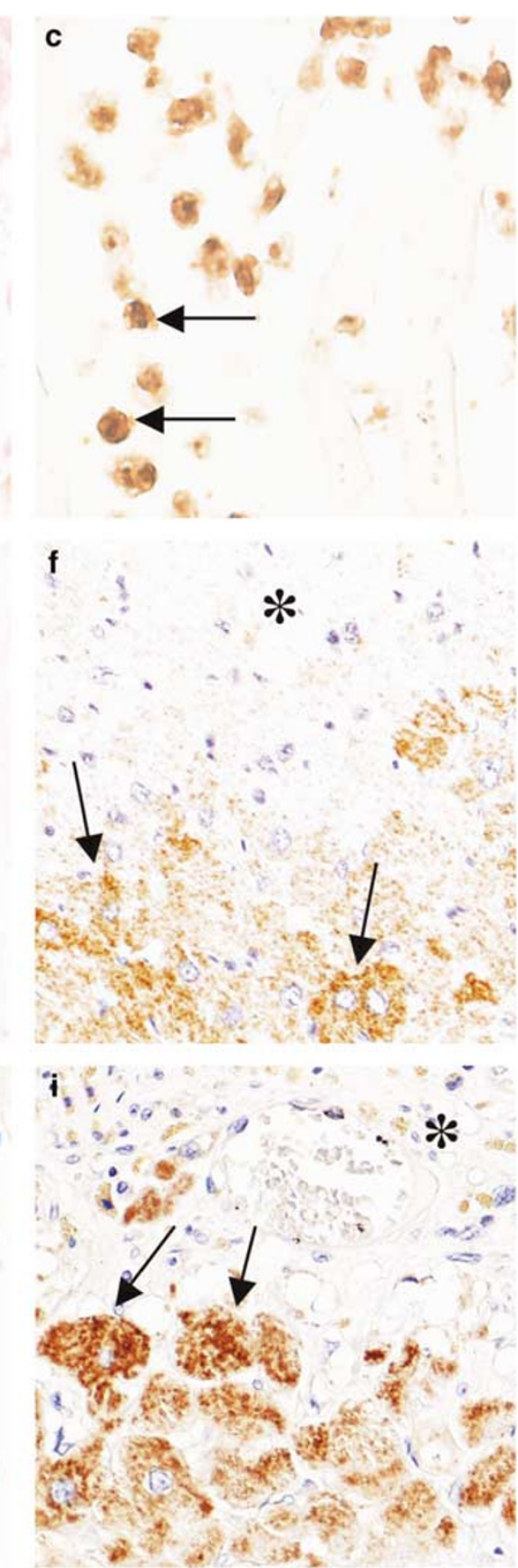

Figure 3 Expression of TNF- $\alpha$, IL-8, and IL-10 at stages I, II, III, IV, and V. (a) IL-8 expression in neutrophils infiltrating the interstitium of a stage I lesion. (b) Double staining of neutrophils at a stage II lesion using antibodies against IL-8 (brown) and myeloperoxidase (red). Arrows indicate infiltrating neutrophils positive for both IL-8 and myeloperoxidase. (c) Control staining included omission of antimyeloperoxidase antibody, and substitution of the antibody by nonspecific rabbit serum. Arrows indicate neutrophils positive for IL8 (brown) only. (d) Controls included omission of anti-IL-8 antibody, and substitution of the antibody by nonspecific rabbit serum. Arrows indicate neutrophils positive for myeloperoxidase (red) only. (e) Controls included omission of myeloperoxidase and IL-8 antibodies, and substitution of the antibodies by nonspecific rabbit serum. The controls show no staining. Hematoxylin staining. (f) TNF$\alpha$ expression in noninfarcted cardiomyocytes (arrows) in the border zone of a stage II lesion. The upper of the figure $(*)$ shows infarcted necrotic cardiomyocytes. (g) Lack of TNF- $\alpha$ expression in the infarcted cardiomyocytes of a stage II lesion. (h) IL-10-positive cells (arrows) in the interstitium around noninfarcted cardiomyocytes in a stage III lesion. (i) TNF- $\alpha$ expression (arrows) in noninfarcted cardiomyocytes in the border zone of a stage III lesion. The upper of the figure $\left({ }^{*}\right)$ shows granulation tissue.

observed in stage II lesions, the expression of TNF- $\alpha$ was predominantly localized in the vicinity of the damaged focus, and strong expression was evident in the border zone (Figure 3i).
In stage IV and V lesions, IL-8-positive neutrophils were absent in both granulatiom tissue and infarct scars. In contrast, TNF- $\alpha$ positivity was evident in a few nondamaged cardiomyocytes in 
the vicinity of the infarct scars or granulation tissue. In addition, IL-8-positive endothelial cells, cardiomyocytes, and macrophages were absent in both infarcted and noninfarcted myocardium at all stages.

\section{Statistical Analysis}

The SI of cardiomyocytes ranged from 0 to $0.47 \%$ (average $0.13 \%$ ), the mean SI increasing rapidly after stage I, peaking at a high level at stage II, and then declining markedly (Figure 4a). Statistical analysis showed that the peak SI at stage II was significantly higher than at stages III, IV, and V (stage III, $P<0.001$; stage IV, $P=0.001$; stage V, $P=0.002)$.
The SI of granulation tissue ranged from 0 to $0.33 \%$ (average $0.14 \%$ ) at stages III, IV, and V, peaking at stage III, and then declining gradually (Figure 4b). No statistically significant difference in the SI was found in granulation tissue cells among the three stages.

The mean TI of cardiomyocytes increased rapidly after stage I, peaked at a high level at stage III, and then declined markedly (Figure 4c). No significant difference in the index was found among the five stages. As TNF- $\alpha$ promotes apoptosis of cultured cardiomyocytes in vitro, we analyzed the correlation between SI and TI in cardiomyocytes, but no significant positive correlation was detected $(r=0.08, P=0.37)$.
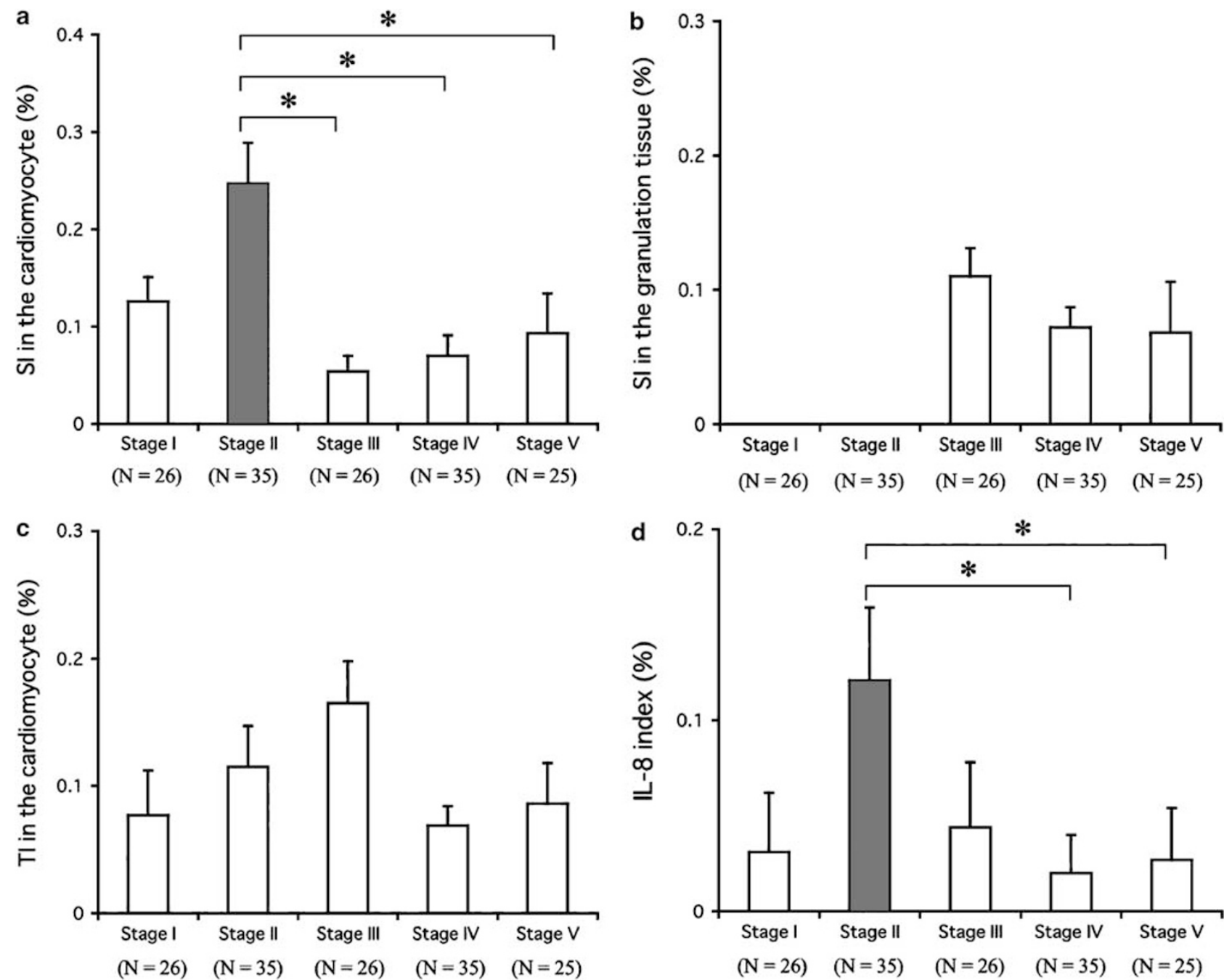

Figure 4 Quantitative analysis of apoptosis, TNF- $\alpha$, and IL-8 at stages I, II, III, IV, and V. (a) The measurement of the ss-DNA index (SI) in cardiomyocytes. The peak SI in cardiomyocytes at stage II was significantly higher than those at stages III, IV, and V (stage III, $P<0.001$; stage IV, $P=0.001$; stage $\mathrm{V}, P=0.002$ ). (b) The measurement of the SI in granulation tissue cells. No significant difference in the SI was found among the three stages. Analysis was not performed in stages I and II, because of lack of the granulation tissue in both stages. (c) The measurement of the TNF- $\alpha$ index (TI) in cardiomyocytes. No significant difference was found in TI among the five stages. (d) The measurement of the IL-8 index in the myocardium. The peak IL-8 index at stage II was significantly higher than the values at stages VI and V (stage IV, $P=0.016$; stage V, $P=0.041$ ). The histogram represents the mean index at each stage. $(N)$ Represents the number of tissue samples examined. ${ }^{*} P<0.05$. 

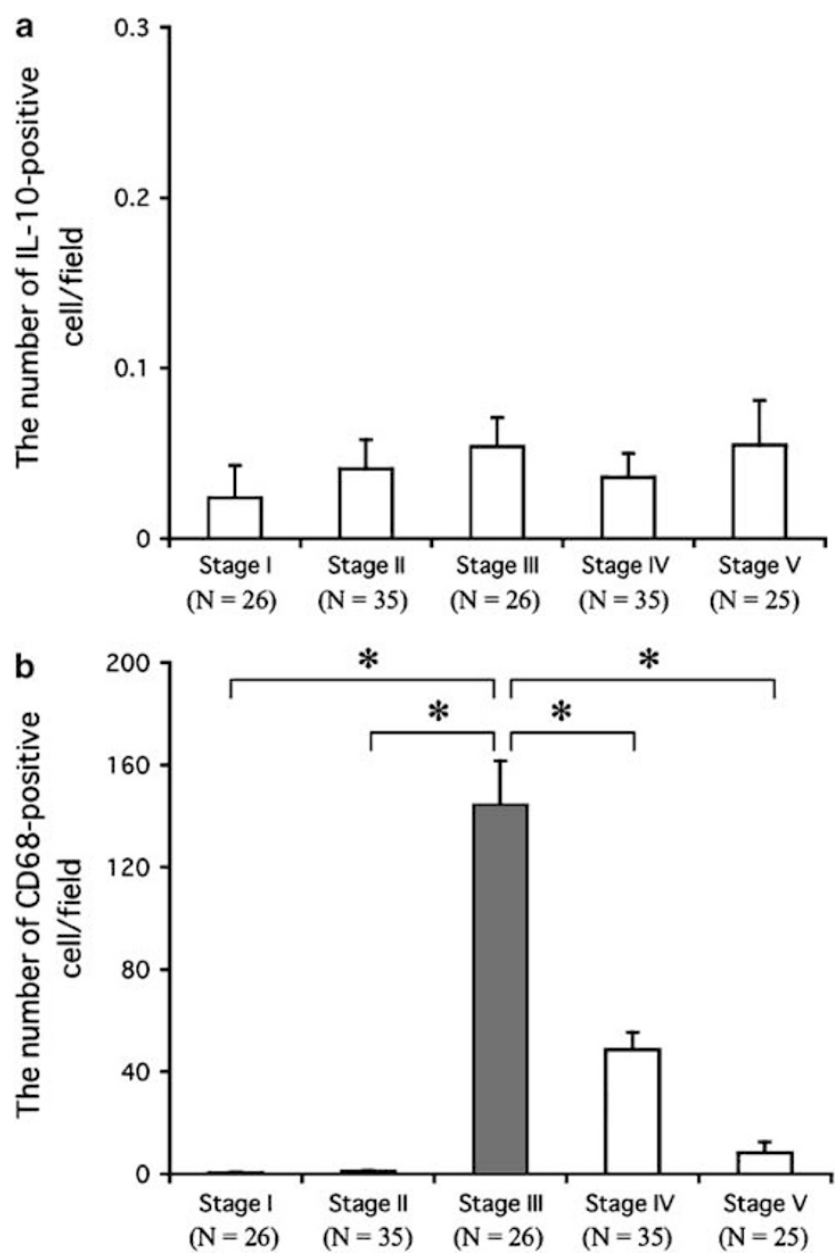

Figure 5 Quantitative analysis of IL-10 and CD-68 expression at stages I, II, III, IV, and V. (a) The measurement of IL-10-positive cells per field in the myocardium. There was no significant difference in the value among the five stages. (b) The measurement of CD-68-positive cells per field in the myocardium. The peak number of CD-68-positive cells per field at stage III was significantly higher than the values at the other four stages (stages I, II, IV, and V, $P<0.001)$. The histogram represents the mean value of positive cells per field at each stage. $(N)$ Represents the number of tissue samples examined. ${ }^{*} P<0.05$.

The mean IL-8 index increased markedly after stage I, peaked at a high level at stage II, and then decreased rapidly from stages II to V (Figure 4d). The peak value at stage II was significantly higher than the values at stages VI and V (stage IV, $P=0.016$; stage $\mathrm{V}, P=0.041$ ).

The mean number of IL-10-positive cells per fields increased sequentially from stage I to stage III, declined slightly at stage IV, and then peaked at stage V (Figure 5a). No significant difference in the index was found among the five stages.

The mean number of CD-68-positive cells per fields increased rapidly from stage II, peaked at stage III, and then declined markedly (Figure 5b). The peak value at stage III was significantly higher than those at the other four stages (stages I, II, IV, and V, $P<0.001)$.

\section{Discussion}

A previous study of human myocardial tissues from autopsy cases using the TUNEL assay demonstrated that $12 \%$ of cardiomyocytes had undergone apoptosis in the border zone of acute MI lesions. ${ }^{10}$ However, in the present study, much lower rates of apoptosis, ranging from $0.13-0.25 \%$, were detected mainly in the border zone at stages II, and the average prevalence of apoptosis at all stages was $0.13 \%$, as demonstrated by staining with the anti-ssDNA mAb. The difference in the proportions of cardiomyocyte apoptosis have been reported in the previous studies, ${ }^{10,29-31}$ and the difference between the studies might have been due to differences in the tissue samples taken or the method used for analysis of myocardial tissue. ${ }^{7,32}$ However, a previous literature by van Empel et al reviewed a prevalence of cardiomyocyte apoptosis ranging from 0.12 to $0.70 \%$ in myocardial biopsy specimens obtained from patients with NYHA classes III-IV heart failure ${ }^{30,33-40}$ which was similar to the figure obtained in the present study. Therefore, the variation in the prevalence of cardiomyocyte apoptosis between the reported studies may not have been due to differences in the myocardial tissue samples used for analysis. ${ }^{10,29}$

In the present study, the peak SI of cardiomyocytes occured at stage II, when the level of cardiomyocyte necrosis was much higher than that at stage I, indicating that apoptosis and necrosis in cardiomyocytes are responsible for acute cellular loss in the acute stage. ${ }^{4}$ As similarly reported in the previous studies, ${ }^{29,41}$ cardiomyocyte apoptosis at stage II was noted in the border zone, indicating that apoptosis mediates cell death outside the area of MI. Furthermore, the peak SI at stage II was significantly higher than at the other three stages. Therefore, cardiomyocyte apoptosis in the border zone occurring specifically in the acute stage suggests that the cell death is involved in cellular loss in the progression of ventricular dysfunction. The induction of apoptosis in the border zone has been suggested to be involved in the left ventricular dilatation. ${ }^{29,41}$ Therefore, cardiomyocyte apoptosis in the border zone might play a significant role in ventricle remodeling during the course of MI.

A recent study showed that inhibtion of apoptosis in granulation tissue cells by a pancaspase inhibitor (Boc-Asp-fmk) improved ventricular remodeling, whereas the level of cardiomyocyte apoptosis was not significantly changed after the treatment. $^{7}$ In contrast, another recent study demonstrated that inhibtion of cardiomyocyte apoptosis by a caspase-3 inhibitor (DEVD-CHO) prevented ventricular dilatation. ${ }^{41}$ The difference in the level of cardiomyocyte apoptosis between the two studies might have been due to the difference in the methods used for inhibition of apoptosis. However, in the present study, the SI of granulation tissue cells showed no significant differences among stages III, IV, and V 
despite the significantly increased SI of cardiomyocytes observed at stage II. A previous study showed that apoptosis remained several months after MI, and hence the previous investigators suggested that myocardial apoptosis in the chronic stage might be a consequence of subsequent ventricular remodeling. ${ }^{41,42}$ Therefore, the lack of stage-specific expression of granulation tissue apoptosis may indicate that it does not play a significant role in cellular loss in the process of ventricular remodeling after MI. Recently, cardiomyocyte apoptosis in mice was found to be well correlated with wall thinning. Inhibition of apoptosis using caspase inhibitor decreased cardiomyocyte apoptosis, with consequent attenuation of wall thinning and ventricular remodeling. ${ }^{43}$ These results suggest that cardiomyocyte apoptosis contributes to ventricular remodeling through wall thinning. In contrast, another study has recently shown that inhibition of apoptosis in granulation tissue in the infarcted region formed thicker walls, which contributed to ventricular remodeling. ${ }^{7}$ Although the time course and type of apoptosis varies in patients with heart failure, the lack of stage-specific expression of granulation tissue apoptosis in the present study indicates that antiapoptotic therapy in patients with heart failure might have profound effects on inhibiting apoptosis in cardiomyocytes, leading to a decrease in ventricular remodeling and heart failure. ${ }^{44}$

In the present study, the temporal and spatial distribution of TNF- $\alpha$-positive cardiomyocyte differed from that of ss-DNA-positive cardiomyocyte; the mean SI of cardiomyocytes peaked at stage II and then declined rapidly, whereas the mean TI peaked at stage III and remained constant well into the later stages, as similarly reported previously. ${ }^{18}$ This difference in timing between SI and TI in cardiomyocytes was confirmed by correlation analysis, which demonstrated no significant relationship between them. Taken together, these results indicate that TNF- $\alpha$ produced by cardiomyocytes may not serve as a direct trigger of cardiomyocyte apoptosis in vivo. ${ }^{13,14,19}$ It has been shown recently that TNF- $\alpha$ activates dual signaling cascades, with one pathway leading to apoptosis, whereas another pathway, mediated through NF- $\kappa \mathrm{B}$, suppresses apoptosis. ${ }^{45}$ Therefore, the latter signal pathway capable of preventing the development of cardiomyocyte apoptosis may play an important role in TNF- $\alpha$ signaling in the infarcted myocardium in vivo. ${ }^{46,47}$

IL-8 is produced by numerous cells including fibroblasts, endothelial cells, lymphocytes, neutrophils, and macrophages..$^{22,48-52}$ In the present study, the levels of IL-8 expression and cardiomyocyte apoptosis peaked at the same stage (stage II), but the cardiomyocytes that underwent apoptosis showed no IL-8 expression. Furthermore, the number of CD68-positive macrophages peaked at the later stage of MI (stage III) and showed no IL-8 expression. These results suggest that human macrophages and cardiomyocytes are not responsible for production of IL-8 in response to apoptotic change. The predominant source of $\mathrm{IL}-8$ is thought to be endothelial cells. ${ }^{25,53}$ However, in the present study, no IL-8 expression was found in any endothelial cells. On the other hand, an unexpected finding was that IL-8 was expressed by neutrophils infiltrating the infarcted myocardium. Although immunohistochemistry can detect intracellular IL-8 only at the protein level, the present finding obviously indicates that infitrating neutrophils are the predominant source of IL-8 in the infarcted myocardium. This is consistent with the previous results showing depletion of circulating leukocytes and loss of IL-8 immunoreactivity in postischemic myocardial tissue. $^{54}$ It is well known that IL-8 is a potent chemoattractant and activator of neutrophils. ${ }^{55,56}$ Therefore, IL-8 produced by neutrophils infiltrating the infarcted myocardium might be responsible for subsequent accumulation and activation of neutrophils, leading to an increase in myocardial cell damage due to the neutrophil-mediated microvascular obstruction. ${ }^{13}$

\section{References}

1 Bialik S, Geenen DL, Sasson IE, et al. Myocyte apoptosis during acute myocardial infarction in the mouse localizes to hypoxic regions but occurs independently of p53. J Clin Invest 1997;100:1363-1372.

2 Fliss H, Gattinger D. Apoptosis in ischemic and reperfused rat myocardium. Circ Res 1996;79:949-956.

3 Gottlieb RA, Burleson KO, Kloner RA, et al. Reperfusion injury induces apoptosis in rabbit cardiomyocytes. J Clin Invest 1994;94:1621-1628.

4 Kajstura J, Cheng W, Reiss K, et al. Apoptotic and necrotic myocyte cell deaths are independent contributing variables of infarct size in rats. Lab Invest 1996;74:86-107.

5 Itoh G, Tamura J, Suzuki M, et al. DNA fragmentation of human infarcted myocardial cells demonstrated by the nick end labeling method and DNA agarose gel electrophoresis. Am J Pathol 1995;146:1325-1331.

6 Saraste A, Pulkki K, Kallajoki M, et al. Apoptosis in human acute myocardial infarction. Circulation 1997; 95:320-323.

7 Hayakawa K, Takemura G, Kanoh M, et al. Inhibition of granulation tissue cell apoptosis during the subacute stage of myocardial infarction improves cardiac remodeling and dysfunction at the chronic stage. Circulation 2003;108:104-109.

8 Takemura G, Fujiwara H. Role of apoptosis in remodeling after myocardial infarction. Pharmacol Ther 2004; 104:1-16.

9 Ottaviani G, Lavezzi AM, Rossi L, et al. Proliferating cell nuclear antigen (PCNA) and apoptosis in hyperacute and acute myocardial infarction. Eur J Histochem 1999;43:7-14.

10 Olivetti G, Quaini F, Sala R, et al. Acute myocardial infarction in humans is associated with activation of programmed myocyte cell death in the surviving portion of the heart. J Mol Cell Cardiol 1996;28: 2005-2016. 
11 Frankfurt OS, Robb JA, Sugarbaker EV, et al. Monoclonal antibody to single-stranded DNA is a specific and sensitive cellular marker of apoptosis. Exp Cell Res 1996;226:387-397.

12 Akishima Y, Akasaka Y, Ishikawa Y, et al. Role of macrophage and smooth muscle cell apoptosis in association with oxidized low-density lipoprotein in the atherosclerotic development. Mod Pathol 2005;18: 365-373.

13 Frangogiannis NG, Smith CW, Entman ML. The inflammatory response in myocardial infarction. Cardiovasc Res 2002;53:31-47.

14 Nian M, Lee P, Khaper N, et al. Inflammatory cytokines and postmyocardial infarction remodeling. Circ Res 2004;94:1543-1553.

15 Levine B, Kalman J, Mayer L, et al. Elevated circulating levels of tumor necrosis factor in severe chronic heart failure. N Engl J Med 1990;323:236-241.

16 McMurray J, Abdullah I, Dargie HJ, et al. Increased concentrations of tumour necrosis factor in 'cachectic' patients with severe chronic heart failure. Br Heart J 1991;66:356-358.

17 Krown KA, Page MT, Nguyen C, et al. Tumor necrosis factor alpha-induced apoptosis in cardiac myocytes. Involvement of the sphingolipid signaling cascade in cardiac cell death. J Clin Invest 1996;98:2854-2865.

18 Irwin MW, Mak S, Mann DL, et al. Tissue expression and immunolocalization of tumor necrosis factoralpha in postinfarction dysfunctional myocardium. Circulation 1999;99:1492-1498.

19 Kubota T, Miyagishima M, Frye CS, et al. Overexpression of tumor necrosis factor- alpha activates both antiand pro-apoptotic pathways in the myocardium. J Mol Cell Cardiol 2001;33:1331-1344.

20 Hughes J, Liu Y, Van Damme J, et al. Human glomerular mesangial cell phagocytosis of apoptotic neutrophils: mediation by a novel CD36-independent vitronectin receptor/thrombospondin recognition mechanism that is uncoupled from chemokine secretion. J Immunol 1997;158:4389-4397.

21 Kurosaka K, Watanabe N, Kobayashi Y. Potentiation by human serum of anti-inflammatory cytokine production by human macrophages in response to apoptotic cells. J Leukoc Biol 2002;71:950-956.

22 Park DR, Thomsen AR, Frevert CW, et al. Fas (CD95) induces proinflammatory cytokine responses by human monocytes and monocyte-derived macrophages. J Immunol 2003;170:6209-6216.

23 Choi C, Kutsch O, Park J, et al. Tumor necrosis factorrelated apoptosis-inducing ligand induces caspasedependent interleukin-8 expression and apoptosis in human astroglioma cells. Mol Cell Biol 2002;22: 724-736.

24 Wan S, DeSmet JM, Barvais L, et al. Myocardium is a major source of proinflammatory cytokines in patients undergoing cardiopulmonary bypass. J Thorac Cardiovasc Surg 1996;112:806-811.

25 Neumann FJ, Ott I, Gawaz M, et al. Cardiac release of cytokines and inflammatory responses in acute myocardial infarction. Circulation 1995;92:748-755.

26 Qi X, Li J, Gu J, et al. Plasma levels of IL-8 predict early complications in patients with coronary heart disease after percutaneous coronary intervention. Jpn Heart J 2003;44:451-461.

27 Lodge-Patch I. The ageing of cardiac infarcts, and its influence on cardiac rupture. Br Heart J 1951;13: $37-42$.
28 Ishikawa Y, Akasaka Y, Ishii T, et al. Changes in the distribution pattern of gelatin-binding protein of $28 \mathrm{kDa}$ (adiponectin) in myocardial remodelling after ischaemic injury. Histopathology 2003;42:43-52.

29 Palojoki E, Saraste A, Eriksson A, et al. Cardiomyocyte apoptosis and ventricular remodeling after myocardial infarction in rats. Am J Physiol Heart Circ Physiol 2001;280:H2726-H2731.

30 Olivetti G, Abbi R, Quaini F, et al. Apoptosis in the failing human heart. $N$ Engl J Med 1997;336: 1131-1141.

31 Gill C, Mestril R, Samali A. Losing heart: the role of apoptosis in heart disease-a novel therapeutic target? FASEB J 2002;16:135-146.

32 Rodriguez M, Schaper J. Apoptosis: measurement and technical issues. J Mol Cell Cardiol 2005;38:15-20.

33 Saraste A, Pulkki K, Kallajoki M, et al. Cardiomyocyte apoptosis and progression of heart failure to transplantation. Eur J Clin Invest 1999;29:380-386.

34 Kang PM, Izumo S. Apoptosis and heart failure: a critical review of the literature. Circ Res 2000;86: 1107-1113.

35 Guerra S, Leri A, Wang X, et al. Myocyte death in the failing human heart is gender dependent. Circ Res. 1999;85:856-866.

36 Narula J, Haider N, Virmani R, et al. Apoptosis in myocytes in end-stage heart failure. N Engl J Med 1996;335:1182-1189.

37 Rayment NB, Haven AJ, Madden B, et al. Myocyte loss in chronic heart failure. J Pathol 1999;188:213-219.

38 Knaapen MW, Davies MJ, De Bie M, et al. Apoptotic versus autophagic cell death in heart failure. Cardiovasc Res 2001;51:304-312.

39 Latif N, Khan MA, Birks E, et al. Upregulation of the Bcl-2 family of proteins in end stage heart failure. J Am Coll Cardiol 2000;35:1769-1777.

40 van Empel VP, Bertrand AT, Hofstra L, et al. Myocyte apoptosis in heart failure. Cardiovasc Res 2005;67: 21-29.

41 Balsam LB, Kofidis T, Robbins RC. Caspase-3 inhibition preserves myocardial geometry and long-term function after infarction. J Surg Res 2005;124:194-200.

42 Chandrashekhar Y, Sen S, Anway R, et al. Long-term caspase inhibition ameliorates apoptosis, reduces myocardial troponin-I cleavage, protects left ventricular function, and attenuates remodeling in rats with myocardial infarction. J Am Coll Cardiol 2004;43: 295-301.

43 Engel D, Peshock R, Armstong RC, et al. Cardiac myocyte apoptosis provokes adverse cardiac remodeling in transgenic mice with targeted TNF overexpression. Am J Physiol Heart Circ Physiol 2004;287: H1303-H1311.

44 Chandrashekhar Y. Role of apoptosis in ventricular remodeling. Curr Heart Fail Rep 2005;2:18-22.

45 Hehlgans T, Mannel DN. The TNF-TNF receptor system. Biol Chem 2002;383:1581-1585.

46 Kurrelmeyer KM, Michael LH, Baumgarten G, et al. Endogenous tumor necrosis factor protects the adult cardiac myocyte against ischemic-induced apoptosis in a murine model of acute myocardial infarction. Proc Natl Acad Sci USA 2000;97:5456-5461.

47 Misra A, Haudek SB, Knuefermann P, et al. Nuclear factor-kappaB protects the adult cardiac myocyte against ischemia-induced apoptosis in a murine model of acute myocardial infarction. Circulation 2003;108: 3075-3078. 
48 Yoshimura T, Matsushima K, Tanaka S, et al. Purification of a human monocyte-derived neutrophil chemotactic factor that has peptide sequence similarity to other host defense cytokines. Proc Natl Acad Sci USA 1987;84:9233-9237.

49 Van Damme J, Decock B, Conings R, et al. The chemotactic activity for granulocytes produced by virally infected fibroblasts is identical to monocyte-derived interleukin 8. Eur J Immunol 1989;19:1189-1194.

50 Schroder JM, Christophers E. Secretion of novel and homologous neutrophil-activating peptides by LPSstimulated human endothelial cells. J Immunol 1989; 142:244-251.

51 Gregory H, Young J, Schroder JM, et al. Structure determination of a human lymphocyte derived neutrophil activating peptide (LYNAP). Biochem Biophys Res Commun 1988;151:883-890.

52 Bazzoni F, Cassatella MA, Rossi F, et al. Phagocytosing neutrophils produce and release high amounts of the neutrophil-activating peptide 1/interleukin 8. J Exp Med 1991;173:771-774.

53 Sica A, Matsushima K, Van Damme J, et al. IL-1 transcriptionally activates the neutrophil chemotactic factor/IL-8 gene in endothelial cells. Immunology 1990;69:548-553.

54 Marx N, Neumann FJ, Ott I, et al. Induction of cytokine expression in leukocytes in acute myocardial infarction. Am Coll Cardiol 1997;30:165-170.

55 Molad Y, Haines KA, Anderson DC, et al. Immunocomplexes stimulate different signalling events to chemoattractants in the neutrophil and regulate L-selectin and beta 2-integrin expression differently. Biochem J 1994;299:881-887.

56 Schroder JM, Mrowietz U, Christophers E. Purification and partial biologic characterization of a human lymphocyte-derived peptide with potent neutrophil-stimulating activity. J Immunol 1988;140: 3534-3540. 